Michael Reiß* ${ }^{*}$ Ady Naber, and Werner Nahm

\title{
Simulating a Ground Truth for Transit Time Analysis of Indicator Dilution Curves
}

https://doi.org/10.1515/cdbme-2018-XXXX

\begin{abstract}
Transit times of a bolus through an organ can provide valuable information for researchers, technicians and clinicians. Therefore, an indicator is injected and the temporal propagation is monitored at two distinct locations. The transit time extracted from two indicator dilution curves can be used to calculate for example blood flow and thus provide the surgeon with important diagnostic information. However, the performance of methods to determine the transit time $\Delta \mathrm{t}$ cannot be assessed quantitatively due to the lack of a sufficient and trustworthy ground truth derived from in vivo measurements. Therefore, we propose a method to obtain an in silico generated dataset of differently subsampled indicator dilution curves with a ground truth of the transit time. This method allows variations on shape, sampling rate and noise while being accurate and easily configurable. COMSOL Multiphysics is used to simulate a laminar flow through a pipe containing blood analogue. The indicator is modelled as a rectangular function of concentration in a segment of the pipe. Afterwards, a flow is applied and the rectangular function will be diluted. Shape varying dilution curves are obtained by discrete-time measurement of the average dye concentration over different cross-sectional areas of the pipe. One dataset is obtained by duplicating one curve followed by subsampling, delaying and applying noise. Multiple indicator dilution curves were simulated, which are qualitatively matching in vivo measurements. The curves temporal resolution, delay and noise level can be chosen according to the requirements of the field of research. Various datasets, each containing two corresponding dilution curves with an existing ground truth transit time, are now available. With additional knowledge or assumptions regarding the detection-specific transfer function, realistic signal characteristics can be simulated. The accuracy of methods for the assessment of $\Delta \mathrm{t}$ can now be quantitatively compared and their sensitivity to noise evaluated.
\end{abstract}

Keywords: indicator dilution curves, ground truth transit time, in silico model

\footnotetext{
*Corresponding author: Michael Reiß, Institute of Biomedical Engineering, Karlsruhe Institute of Technology, Kaiserstrasse 12, Karlsruhe, Germany, e-mail: publications@ibt.kit.edu

Ady Naber, Institute of Biomedical Engineering, Karlsruhe Institute of Technology, Karlsruhe, Germany

Werner Nahm, Institute of Biomedical Engineering, Karlsruhe Institute of Technology, Karlsruhe, Germany
}

\section{Introduction}

In the last two decades, optic-based medical systems were introduced to intraoperatively visualize vascular structures using flourescence angiography $[1,2]$. However, visual inspection may not be sufficient for intraoperative decision making. Therefore, a quantitative approach for intraoperative blood volume flow assessment is needed [2]. A current clinical routine for blood volume flow assessment involves the usage of an ultrasonic flowprobe. This clinical flowprobe has an accuracy of $\pm 10 \%$ [3], but downsides are additional time, equipment and interrupting surgical workflow; compromising the vessel due to mechanical stress is possible as well [4]. Thus, a method without tissue contact would be advantageous. For such a non-invasive method, intraoperative Fluorescence Angiography can be used. Therefore, an indicator bolus is injected into the vascular system. Afterwards, two indicator dilution curves (IDCs) are obtained by monitoring the temporal propagation of the indicator bolus at two distinct locations along the vessel of interest. The transit time of the bolus can be extracted from those two IDCs. The blood volume flow $\dot{V}$ can subsequently be calculated as shown in Eq. 1, where $A$ is the vessel's cross-sectional area, $v$ the blood flow velocity, $d_{i}$ the inner diameter of the vessel and $\Delta t$ the transit time of the indicator bolus to travel the distance $s$.

$$
\dot{V}=A \cdot v=\frac{\pi \cdot d_{i}^{2} \cdot s}{4 \cdot \Delta t}
$$

Although in vivo IDCs are monitored frequently in clinical routines and are thus widely available [5], they lack a sufficient and trustworthy ground truth of the transit time. By simply duplicating and delaying an in vivo IDC, a credible performance evaluation of methods ascertaining the transit time $\Delta \mathrm{t}$ via two IDCs cannot be assured due to the usage of identical data points for both corresponding curves.

Therefore, we propose a method to synthetically generate a highly adaptable dataset of two corresponding and differently subsampled IDCs with a ground truth of the transit time using an in silico model. These datasets can then be used to enhance current methods ascertaining the transit time $\Delta \mathrm{t}$, which temporal accuracies are not yet suitable for clinical studies [6]. Even though an in vitro flow phantom could provide a similar dataset, in silico generated datasets are low-cost, accurate and easily configurable to the desired field of application [7]. 


\section{Methods}

The method chapter is structured into two parts. The first part describes the dilution curve data acquisition using a deterministic in silico model of a laminar flow through a rigid pipe containing blood analogue solution. The second part describes the post-processing steps to obtain the desired dataset of two corresponding IDCs with a ground truth of the transit time.

\subsection{In silico data acquisition}

To simulate the in silico model, the finite element based software COMSOL Multiphysics (version 5.4) with the additional packages 'Computational Fluid Dynamics' and 'Chemical Reaction Engineering' was used. The rotationally symmetrical in silico model was devided into a setup-section containing blood analogue and the indicator bolus, and a measurement-section to later monitor the indicator bolus' concentration. The following boundary conditions and simplifications were defined and allow the reproducibility of the results:

- Applied volume flow: $\dot{V}=150 \frac{\mathrm{ml}}{\mathrm{min}}$

- Inner radius of the pipe: $r_{i}=2 \mathrm{~mm}$

- Length of the setup-section: $1.2 \mathrm{~m}$

- Length of the measurement-section: $1 \mathrm{~m}$

- Incompressible fluid: $\rho=$ const.

- Laminar fluid flow and convection: $R e<R e_{\text {crit }}$

- Rigid vessel walls: $E=0$

- No-slip: $u\left(r=r_{i}\right)=0$

- Isothermal process: $\Delta K=0$

- No external or hydrostatic pressure

- No gravitational force

- Non-Newtonian fluid behaviour

The blood analogue solution containing demineralized water, glycerol, xanthan and protein was set to a density of $1157 \frac{\mathrm{kg}}{\mathrm{m}^{3}}$ and a viscosity of $11.64 \mathrm{mPa} \cdot \mathrm{s}$. The injection of the indicator bolus was modelled as a rectangular function of the indicator concentration midway in the pipe's setup-section. After the initialization of the in silico model was finished, a laminar flow was applied to the blood analogue solution resulting in the dilution of the rectangular function across the pipe. To obtain a time-discrete dilution curve, the average indicator concentration was monitored over the cross-sectional area of the pipe in the measurement-section. The timespan of the measurement was set to monitor the dilution curve in it's entirely, the temporal resolution was chosen to $1200 \mathrm{~Hz}$ as a trade-off between achieved high temporal resolution and computing time. As shown in Fig. 1, changing the location in the measurementsection allowed a variation of the dilution curve's shape.

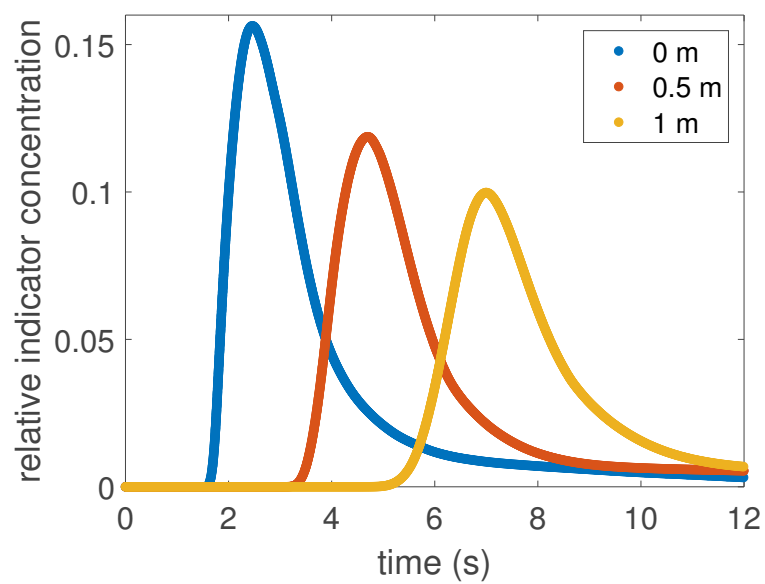

Fig. 1: Shape varying IDCs of relative indicator concentrations measured at the positions $0 \mathrm{~m}, 0.5 \mathrm{~m}$ and $1 \mathrm{~m}$ from the begin of the measurement-section. The timespan is set to 12 seconds and the temporal resolution is set to $1200 \mathrm{~Hz}$.

\subsection{Dataset creation}

After one discrete-time IDC was acquired, the dataset of two corresponding IDCs could be created by either using two shape varying curves or by duplicating one single dilution curve. Since the dispersion of the indicator bolus between the two measurement locations along the vessel of interest is minimal and therefore negligible, we followed the approach to generate the dataset by duplicating one single dilution curve. Afterwards, the temporal delay as the ground truth transit time was applied to one of the duplicated curves followed by differently subsampling and noising the dataset to qualitatively match in vivo measurements. The single steps will be described in detail in the following section, all post-processing was done using MATLAB R2019b.

\subsubsection{Temporal delay with ground truth transit time}

After duplicating one acquired dilution curve, the temporal delay was applied to one of the two IDCs using Eq. 2, where $\left\{t_{1}, \ldots, t_{n}\right\}$ are the $n$ timestamps of the duplicated IDC and $\left\{t_{d 1}, \ldots, t_{d n}\right\}$ are the timestamps after application of the temporal delay $\Delta \mathrm{t}$.

$$
\left\{t_{d 1}, \ldots, t_{d n}\right\}=\left\{t_{1}+\Delta t, \ldots, t_{n}+\Delta t\right\} \forall n \in \mathbb{N}
$$

The thereby provided ground truth of the transit time $\Delta \mathrm{t}$ was chosen to match a suspected physiological transit time of the indicator bolus between two corresponding in vivo dilution curves. Fig. 2 shows a dataset created by duplicating one IDC from the in silico model and application of the temporal delay.

\subsubsection{Subsampling}

After applying the temporal delay, the resulting two curves were subsampled. This allowed the adaption of the two in sil- 


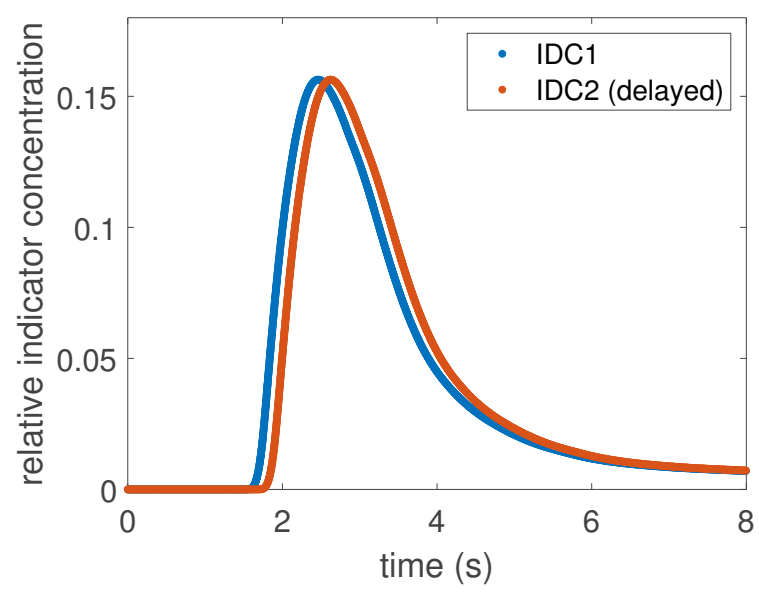

Fig. 2: Duplicated IDCs $(1200 \mathrm{~Hz})$ with an arbitrarily chosen ground truth transit time of $\Delta t=0.16 \mathrm{~s}$ applied to IDC2.

ico generated curves to the temporal resolution of different surgical systems. Due to the acquisition of an in silico dataset with high temporal resolution from COMSOL Multiphysics, different subsamples of identical temporal resolutions could be created, as shown in Fig. 3, and used for further processing.

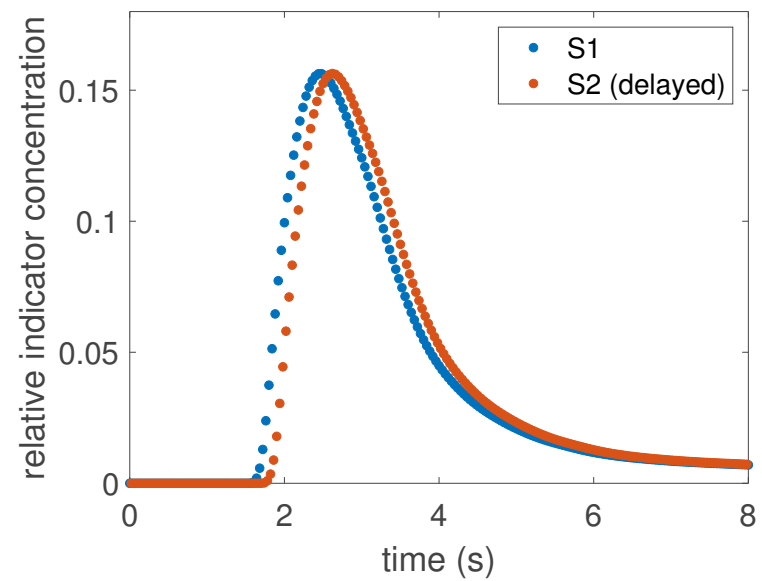

Fig. 3: Differently subsampled IDCs (S1, S2) of Fig. 2 to a temporal resolution of $25 \mathrm{~Hz}$.

\subsubsection{Noise application}

A dataset with a known ground truth transit time has been created. However, noise is unavoidable in data acquisition and therefore applied to the dataset to match IDCs obtained from in vivo measurements. We propose the application of additive white gaussian noise due to its property to represent random sources of thermal noise by adding independent values to each discrete-time data point [8]. The noise level is described via the signal-to-noise ratio (SNR), as shown in Eq. 3, where $P_{\text {signal }}$ is the power of the signal and $P_{\text {noise }}$ is the power of the noise. Due to the wide dynamic range of noise levels, the SNR is expressed using the logarithmic decibel scale.

$$
S N R=10 \log \left(\frac{P_{\text {signal }}}{P_{\text {noise }}}\right) d B
$$

Fig. 4 illustrates an example of a dataset after noise application.

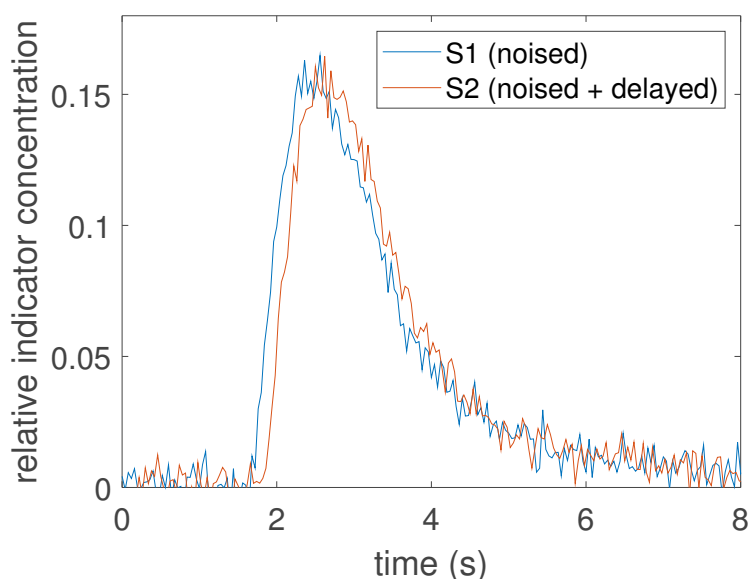

Fig. 4: Application of noise to the curves of Fig. 3, each with an arbitrarily chosen SNR of $20 \mathrm{~dB}$. Linear connections are added between the discrete-time data.

\section{Results}

Multiple datasets of two corresponding IDCs with differently subsampled data points and a ground truth of the transit time were simulated. The synthetically generated curves of a dataset, as shown in Fig. 6, qualitatively match clinically obtained in vivo measurements (Fig. 5) before recirculation occurs. Regarding different fields of research, the in silico curves can be adjusted accordingly by altering the curves shape, the temporal resolutions, the subsampling, the ground truth of the transit time and the noise level.

\section{Discussion and Conclusion}

Obtaining the ground truth transit time of indicator dilution curves is a challenge. We have proposed and implemented a method to generate datasets of two corresponding IDCs, allowing a high degree of freedom varying the ground truth transit time $\Delta t$ as well as the signals' morphology, sampling rate and noise level. Although the presented dilution curves were simulated by an in silico model using an optical dye indicator (particle concentration), the model should be also transferable to simulate various indicator types such as thermal indicators (with a non-isothermal in silico model). This allows a versatile use of the model to simulate different recording systems or signals obtained from different organs. However, the proposed in silico model is only valid if a laminar fluid flow in rigid vessels can be assumed. Further, this in silico model represents the blood vessel as a straight rigid pipe, limiting the credibility of data points on the curve's descending side where recirculation is not modelled. Nevertheless, in most methods ascertaining the transit time $\Delta \mathrm{t}$, the dilution curve is cut off 


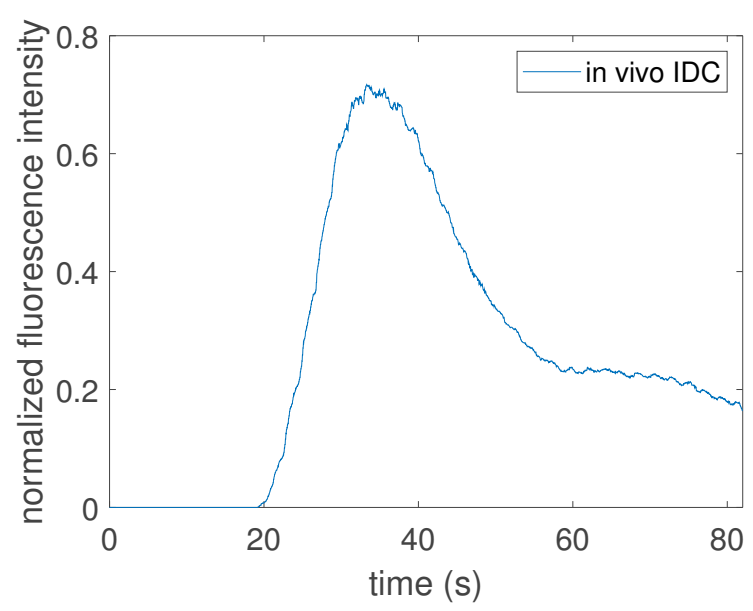

Fig. 5: In vivo IDC with a temporal resolution of $30 \mathrm{~Hz}$, monitored using intraoperative Fluorescence Angiography.

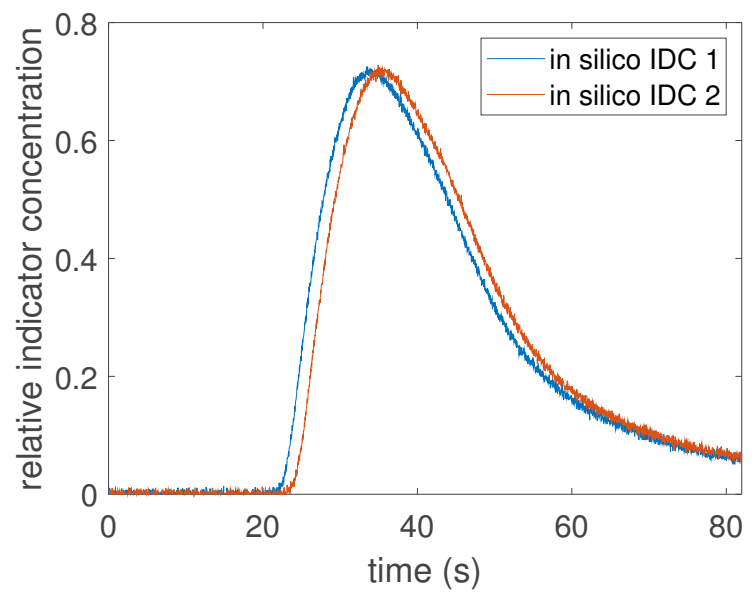

Fig. 6: Synthetically generated dataset of two corresponding IDCs. The curves are differently subsampled to $30 \mathrm{~Hz}$ and noised with a SNR of $37 \mathrm{~dB}$. A temporal delay of $\Delta t=1.5 \mathrm{~s}$ is applied to IDC 2.

after its concentration had decreased to $50 \%$ of the respective peak, which excludes recirculation [5]. To represent random sources of thermal noise, we applied white gaussian noise to the datasets' two corresponding curves. However, the simplification to only take thermal sources of noise into account may not be sufficient since various nonthermal sources of noise and/or artifacts can also be observed in clinical in vivo measurements. A generalized and quantitative comparison of the in silico generated curves with clinical data obtained from intraoperative fluorescence angiography is not trivial due to the diversity of possible morphologies and fields of application. However, a qualitative comparison of the simulated and the measured signals shows a high accordance.

\section{Outlook}

Various datasets, each containing two corresponding dilution curves with an existing ground truth transit time, are now available for further adaptations or analysis. With additional knowledge or assumptions regarding the detectionspecific transfer function, application tailored signal characteristics can be derived and implemented. The accuracy of new methods ascertaining the transit time $\Delta t$ between two IDCs can now be evaluated regarding their sensitivity to the signals' morphology, sampling rate and/or noise by comparison to the provided ground truth transit time. Thereby, no model-based restriction in the methods selection is necessary.

Acknowledgment: We thank Priv. Doz. Dr. med. Uwe Spetzger of the Städtisches Klinikum Karlsruhe for the support by providing videos of neurovascular interventions.

\section{Author Statement}

Research funding: The research is funded by the Carl Zeiss Meditec AG. Conflict of interest: Authors state no conflict of interest. Informed consent: Informed consent has been obtained from all individuals included in this study. Ethical approval: The research related to human use complies with all the relevant national regulations, institutional policies and was performed in accordance with the tenets of the Helsinki Declaration, and has been approved by the partners' collaborative institutional review board or equivalent committee.

\section{References}

[1] Kamp M. A, Slotty, P, Turowski B, Etminan N, Steiger H. J, Hänggi $D$, et al. Microscope-integrated quantitative analysis of intraoperative indocyanine green fluorescence angiography for blood flow assessment: first experience in $30 \mathrm{pa}$ tients. Operative Neurosurgery 2012;70:65-74.

[2] Raabe A, Beck J, Gerlach R, Zimmermann M, Seifert V. Near-infrared indocyanine green video angiography: a new method for intraoperative assessment of vascular flow. Neurosurgery 2003; 52(1):132-139.

[3] Transonic. Transonic Clinical Flowprobes manual 2019.

[4] Amin-Hanjani S, Meglio G, Gatto R, Bauer A, Charbel F T. The utility of intraoperative blood flow measurement during aneurysm surgery using an ultrasonic perivascular flow probe. Operative Neurosurgery 2006;58:0-305.

[5] Reuter D. A, Huang C, Edrich T, Shernan S. K, Eltzschig H. K. Cardiac output monitoring using indicator-dilution techniques: basics, limits, and perspectives. Anesthesia \& Analgesia 2010;110(3):799-811.

[6] Cimalla P, Graf D, Duscha P, Meyer T, Kuß J, Steinmeier $R$, et al. Methods for determining the blood flow velocity in cerebral vessels using intraoperative Indocyanine Green fluorescence video angiography. 4th European Conference of the International Federation for Medical and Biological Engineering 2009, Springer, Berlin, Heidelberg:1446-1449

[7] Kung E. O, Les A. S, Figueroa C. A, Medina F, Arcaute K, Wicker R. B, Taylor C. A In vitro validation of finite element analysis of blood flow in deformable models. Annals of biomedical engineering 2011;39(7):1947-1960.

[8] Sakai H. M. White-noise analysis in neurophysiology. Physiological Reviews 1992;72(2):491-505. 